\title{
Large Deviation Result for the Empirical Locality Measure of Typed Random Geometric Graphs
}

\author{
Kwabena Doku-Amponsah
}

Correspondence: Kwabena Doku-Amponsah, Statistics Department, School of Physical and Mathematical Sciences, College of Basic and Applied Sciences, University of Ghana, Box LG115, Legon, Ghana.

E-mail: kdoku@ug.edu.gh.

Received: November 11, 2014 Accepted: November 30, 2014 Online Published: January 12, 2015

doi:10.5539/ijsp.v4n1p94 URL: http://dx.doi.org/10.5539/ijsp.v4n1p94

\begin{abstract}
In this article for a finite typed random geometric graph we define the empirical locality distribution, which records the number of nodes of a given type linked to a given number of nodes of each type. We find large deviation principle (LDP) for the empirical locality measure given the empirical pair measure and the empirical type measure of the typed random geometric graphs. From this LDP, we derive large deviation principles for the degree measure and the proportion of detached nodes in the classical Erdős-Rényi graph defined on $[0,1]^{d}$. This graphs have been suggested by (Canning and Penman, 2003) as a possible extension to the randomly typed random graphs.
\end{abstract}

Keywords: typed random geometric graph, empirical locality measure, empirical degree measure, detached nodes

Mathematics Subject Classification: 60F10, 05C80.

\section{Introduction}

The typed random geometric graph (TRGG) is obtain when $n$ nodes or nodes or points are placed uniformly at random in $[0,1]^{d}$, and in addition each node is assigned an independently chosen type or colour or symbol or spin from a finite alphabet $\mathcal{B}$. And any two points with types $a_{1}, a_{2}$ (resp.) distance at most $r_{n}\left(a_{1}, a_{2}\right)$ apart are linked. The linking radius $r_{n}$ plays similar role as the link probability $p_{n}$ in the randomly coloured random graph models introduced by (Penman, 1998), surveyed by (Canning and Penman, 2003) and studied in (Doku-Amponsah and Moerters, 2010). The classical Erdős-Rényi graph on $[0,1]^{d}$ is obtain when $n$ points are chosen at random uniformly and independently from $[0,1]^{d}$ and $\lambda_{n}$ edges are inserted at random among the nodes.

In this article we extend the LDP for the empirical locality measure conditioned on the empirical pair measure and the empirical type distribution, see (Doku-Amponsah et al., 2010, Theorem 2.5), to TRGG models. From this result we derive the LDP for the degree distribution and proportion of detached nodes the classical Erdôs-Rényi graph defined on $[0,1]^{d}$. See (Doku-Amponsah, 2014) for similar result for the classical Erdö-Renyi graphs.

Note that the LDP for the empirical locality measure conditioned on a given empirical pair measure and empirical type measure of TRGG is a crucial step in the establishment of a full large deviation principle for the empirical locality measure of TRGG. See (Doku-Amponsah, 2014[b]).

\subsection{TRGG Model}

The TRGG is a general model of random geometric graphs in which the linking radius depends on the type or type of the nodes. The main statistics for this model of random graphs are the empirical pair distribution and the empirical type distribution.

Given a probability measure $v$ on $\mathcal{B}$ and a symmetric function $r_{n}: \mathcal{B} \times \mathcal{B} \rightarrow(0,1]$ we define the randomly typed random geometric graph or typed random geometric graph $X$ having $n$ nodes as follows: Pick nodes $X_{1}, \ldots, X_{n}$ at random independently according to the uniform distribution on $[0,1]^{d}$. We assign to each node $X_{j}$ type $Z\left(X_{j}\right)$ at random and independently according to the type law $v$. Given the types, we link any two nodes $X_{i}, X_{j},(i \neq j)$ by an edge independently of everything else, if

$$
\left\|X_{i}-X_{j}\right\| \leq r_{n}\left[Z\left(X_{i}\right), Z\left(X_{j}\right)\right]
$$


In this article we shall refer to $r_{n}\left(a_{1}, a_{2}\right)$, for $a_{1}, a_{2} \in \mathcal{B}$ as a link radius, and always look at

$$
\left.X=\left(\left(Z\left(X_{i}\right), Z\left(X_{j}\right)\right): i, j=1,2,3, \ldots, n\right), E\right)
$$

under the joint measure of graph and type. We look at $X$ as TRGG with nodes $X_{1}, \ldots, X_{n}$ chosen at random uniformly and independently from the nodes space $[0,1]^{d}$. For the purposes of this study we restrict ourselves to the near intermediate cases .i.e. the link radius $r_{n}$ satisfies the condition $n r_{n}^{d}\left(a_{1}, a_{2}\right) \rightarrow \lambda\left(a_{1}, a_{2}\right)$ for all $a_{1}, a_{2} \in \mathcal{B}$, where $\lambda: \mathcal{B}^{2} \rightarrow[0, \infty)$ is a symmetric function, which is not identically equal to zero.

For any set of finite or countable elements $\mathcal{B}$, let $\mathcal{P}(\mathcal{B})$ be the space of probability vectors, and $\tilde{\mathcal{P}}(\mathcal{B})$ the space of finite vectors on $\mathcal{B}$, both equipped with the weakest topology on $\mathcal{P}(\mathcal{B})$. By convention we write

$$
\mathcal{Z}=\{0,1,2, \ldots\}
$$

We associate with any typed graph $X$ a probability measure, the empirical type distribution $\mathcal{L}_{X}^{1} \in \mathcal{P}(\mathcal{B})$, by

$$
\mathcal{L}_{X}^{1}(a):=\frac{1}{n} \sum_{j=1}^{n} \delta_{Z\left(X_{j}\right)}(a), \quad \text { for } a_{1} \in \mathcal{B},
$$

and the empirical pair measure $\mathcal{L}_{X}^{2} \in \tilde{\mathcal{P}}_{*}\left(\mathcal{B}^{2}\right)$, by

$$
\mathcal{L}_{X}^{2}\left(a_{1}, a_{2}\right):=\frac{1}{n} \sum_{(i, j) \in E}\left[\delta_{\left(X\left(Y_{i}\right), Z\left(X_{j}\right)\right)}+\delta_{\left(\left(Z\left(X_{j}\right), X\left(Y_{i}\right)\right)\right.}\right]\left(a_{1}, a_{2}\right), \quad \text { for }(a, b) \in \mathcal{B}^{2} .
$$

Also we define the empirical locality measure $\mathcal{M}_{X} \in \mathcal{P}(\mathcal{B} \times \mathcal{Z})$, by

$$
\mathcal{M}_{X}\left(a_{1}, \sigma\right):=\frac{1}{n} \sum_{j=1}^{n} \delta_{\left(Z\left(X_{j}\right), \mathcal{A}\left(X_{j}\right)\right)}\left(a_{1}, \sigma\right), \quad \text { for }\left(a_{1}, \sigma\right) \in \mathcal{B} \times \mathcal{Z},
$$

where $\mathcal{A}(i)=\left(\sigma^{i}\left(a_{2}\right), a_{2} \in \mathcal{B}\right)$ and $\sigma^{i}(b)$ is the number of nodes of type $a_{2}$ linked to node $i$.

For any $n \in \mathcal{Z}$ we define

$$
\begin{aligned}
\mathcal{P}_{n}(\mathcal{B}) & :=\{\varpi \in \mathcal{P}(\mathcal{B}): n \varpi(a) \in \mathcal{Z} \text { for all } a \in \mathcal{B}\}, \\
\tilde{\mathcal{P}}_{n}(\mathcal{B} \times \mathcal{B}) & :=\left\{\omega \in \tilde{\mathcal{P}}_{*}(\mathcal{B} \times \mathcal{B}): \frac{n}{1+\mathbb{1}\left\{a_{1}=a_{2}\right\}} \omega\left(a_{1}, a_{2}\right) \in \mathcal{Z} \text { for all } a_{1}, a_{2} \in \mathcal{B}\right\},
\end{aligned}
$$

\subsection{Conditional TRGG.}

Let $\varpi\left(a_{1}\right)>0$, for all $a_{1} \in \mathcal{B}$. We observe that the distribution of the TRGG if the empirical type measure $\varpi_{n}$ and empirical pair distribution $\omega_{n}$,

$$
\mathbb{P}_{\left(\varpi_{n}, \omega_{n}\right)}:=\mathbb{P}\left\{\cdot \mid \mathcal{H}\left(\mathcal{M}_{X}\right)=\left(\varpi_{n}, \omega_{n}\right)\right\},
$$

may be obtained as follows:

- Pick nodes $X_{1}, \ldots, X_{n}$ at random independently according to the uniform distribution on $[0,1]^{2}$.

- Give types to the nodes by picking without replacement from the constellation of $n$ types, containing each type $a_{1} \in \mathcal{B}$ precisely $n \varpi_{n}(a)$ times;

- For each pair $\left\{a_{1}, a_{2}\right\}$ of types make precisely $n\left(a_{1}, a_{2}\right)$ links by picking without replacement from the pool of potential edges linking nodes of type $a_{1}$ and $a_{2}$, where

$$
m_{n}\left(a_{1}, a_{2}\right):= \begin{cases}n \omega_{n}\left(a_{1}, a_{2}\right) & \text { if } a_{1} \neq a_{2} \\ \frac{n}{2} \omega_{n}\left(a_{1}, a_{2}\right) & \text { if } a_{1}=a_{2}\end{cases}
$$

In the remainder of the paper we state and prove our LDP results. In Section 0.1 we state our LDPs, Theorem 0.1, Theorem 0.2, and Corollary 0.3. The proof of Theorem 0.1, carried out in Section 0.1, uses a combinatorial arguments based on random allocation of typed balls into typed bins and (Doku-Amponsah,Lemma 5, 2014). The article ends with the proofs of Theorem 0.2 and 0.3 in Subsection 0.1. 


\section{Statement of the Results}

For any $\ell \in \mathcal{P}\left(\mathcal{B} \times \mathcal{Z}^{\mathcal{B}}\right)$ we denote by $\ell_{1}$ the $\mathcal{B}$ - marginal of $\ell$ and for every $\left(a_{2}, a_{1}\right) \in \mathcal{B} \times \mathcal{B}$, let $\ell_{2}$ be the distribution of the couple $\left(a_{1}, \sigma\left(a_{2}\right)\right)$ under the measure $\ell$. We define the finite measure, $\mathcal{H} \in \tilde{\mathcal{P}}(\mathcal{B} \times \mathcal{B})$ by

$$
\mathcal{H}_{2}(\ell)\left(a_{2}, a_{1}\right):=\sum_{\sigma\left(a_{2}\right) \in \mathcal{Z}} \ell_{2}\left(a_{1}, \sigma\left(a_{2}\right)\right) \sigma\left(a_{2}\right), \quad \text { for } a_{1}, a_{2} \in \mathcal{B}
$$

and write $\mathcal{H}_{1}(\ell)=\ell_{1}$. We define the function $\mathcal{H}: \mathcal{P}\left(\left(\mathcal{B} \times \mathcal{Z}^{\mathcal{B}}\right) \rightarrow \mathcal{P}(\mathcal{B}) \times \tilde{\mathcal{P}}(\mathcal{B} \times \mathcal{B})\right.$ by $\mathcal{H}(\ell)=\left(\mathcal{H}_{1}(\ell), \mathcal{H}_{2}(\ell)\right)$ and observe that $\mathcal{H}\left(\mathcal{M}_{X}\right)=\left(\mathcal{L}_{X}^{1}, \mathcal{L}_{X}^{2}\right)$. Note that, in the weak topology $\mathcal{H}_{1}$ is not discontinuous function but $\mathcal{H}_{2}$ is. To be specific, in the expression $\sum_{\sigma\left(a_{2}\right) \in \mathcal{Z}} \ell_{2}\left(a_{1}, \sigma\left(a_{2}\right)\right) \sigma\left(a_{2}\right)$ the function $\sigma\left(a_{2}\right)$ may not be bounded and hence in the weak topology the functional $\ell \rightarrow \mathcal{H}_{2}(\ell)$ would be discontinuous. We say two of measures $(\omega, \ell) \in \tilde{\mathcal{P}}(\mathcal{B} \times \mathcal{B}) \times \mathcal{P}\left(\mathcal{B} \times \mathcal{Z}^{\mathcal{B}}\right)$ consistent if

$$
\mathcal{H}_{2}(\ell)\left(a_{2}, a_{1}\right)=\omega\left(a_{2}, a_{1}\right), \quad \text { for all } a_{1}, a_{2} \in \mathcal{B} .
$$

The next theorem gives LDP for the empirical locality measure of a sequence of graphs with given empirical type distribution and empirical pair distribution.

Theorem 0.1. Let the sequence $\left(\varpi_{n}, \omega_{n}\right) \in \mathcal{P}_{n}(\mathcal{B}) \times \tilde{\mathcal{P}}_{n}(\mathcal{B} \times \mathcal{B})$ converges to a limit $\left((\varpi, \omega) \in \mathcal{P}(\mathcal{B}) \times \tilde{\mathcal{P}}_{*}(\mathcal{B} \times \mathcal{B})\right.$. Suppose that $X$ is a TRGG graph conditioned on the set $\left\{\mathcal{H}\left(\mathcal{M}_{X}\right)=\left(\varpi_{n}, \omega_{n}\right)\right\}$. Then, the empirical locality measure $\mathcal{M}_{X}$, as $n \rightarrow \infty$, satisfies an $L D P$ in the space $\mathcal{P}(\mathcal{B} \times \mathcal{Z})$ with good rate function

$$
\tilde{J}_{((\varpi, \omega)}(\ell)= \begin{cases}H\left(\ell \| Q_{p o i}\right) & \text { if }(\omega, \ell) \text { is consistent and } \ell_{1}=\varpi_{2} \\ \infty & \text { otherwise. }\end{cases}
$$

where

$$
Q_{p o i}\left(a_{1}, \sigma\right)=\ell_{1}\left(a_{1}\right) \prod_{a_{2} \in \mathcal{B}} e^{-\frac{\omega\left(a_{1}, a_{2}\right)}{\ell_{1}\left(a_{1}\right)}} \frac{1}{\sigma\left(a_{2}\right) !}\left(\frac{\omega\left(a_{1}, a_{2}\right)}{\ell_{1}(a)}\right)^{\sigma\left(a_{2}\right)}, \quad \text { for } a_{1} \in \mathcal{B}, \sigma \in \mathcal{Z} .
$$

Note that degree distribution $D_{X} \in \mathcal{P}(\mathcal{Z})$ of a graph with empirical locality distribution $\mathcal{M}_{X}$ is given by

$$
D_{X}(r)=\sum_{a_{1} \in \mathcal{B}} \sum_{\sigma \in \mathcal{Z}} \delta_{r}\left(\sum_{a_{2} \in \mathcal{B}} \sigma\left(a_{2}\right)\right) \mathcal{M}_{X}\left(a_{1}, \sigma\right), \quad \text { for } r \in \mathcal{Z},
$$

i.e. $D_{X}(r)$ is the proportion of nodes in the graph with degree $r$. Theorem 0.2 below is a spacial case of Theorem 0.1 above where $\mathcal{M}_{X}=D_{X}$, the degree distribution and $\left\langle\mathcal{H}\left(\mathcal{M}_{X}\right)\right\rangle=2|E| / n$.

We write

where $\Gamma$ is the gamma function.

$$
\rho(d)=\frac{\pi^{d / 2}}{\Gamma\left(\frac{(d+2)}{2}\right)},
$$

Theorem 0.2. Suppose the sequence $\lambda_{n} / n$ converges to a limit $\rho(d) t / 2$. Let $\mathcal{G}\left(n, \lambda_{n}\right)$ be a Random geometric graph, where nodes $X_{1}, \ldots, X_{n}$ are chosen at random uniformly and independently from $[0,1]^{d}$, and $\lambda_{n}$ edges are inserted at random among the nodes. Then, as $n \rightarrow \infty$, the degree distribution $D_{X}$ of $\mathcal{G}\left(n, \lambda_{n}\right)$ satisfies large deviation principle on the space $\mathcal{P}(\mathcal{Z})$ with good rate function

$$
\eta(\delta)= \begin{cases}H\left(\delta \| q_{\langle\delta\rangle}\right), & \langle\delta\rangle=\rho(d) t \\ \infty & \text {, otherwise }\end{cases}
$$

From Theorem 0.2 above we obtain the following Corollary 0.3 using the contraction principle. See (Dembo and Zeitouni, 1998).

Corollary 0.3. Suppose the sequence $\lambda_{n} / n$ converges to a limit $\rho(d) t / 2$. Let $\mathcal{G}\left(n, \lambda_{n}\right)$ be a random geometric graph, where nodes $X_{1}, \ldots, X_{n}$ are chosen at random uniformly and independently from $[0,1]^{d}$, and $\lambda_{n}$ edges are inserted at random among the nodes. Then, the proportion of detached nodes $D_{X}(0)$ of $\mathcal{G}\left(n, \lambda_{n}\right)$, as $n \rightarrow \infty$, obeys an LDP on the space $[0,1]$ with good, convex rate function

$$
\xi(y)=\left\{\begin{array}{lr}
y \log \frac{y}{1-e^{-\rho(d) t}}+(1-y) \log \frac{(1-y)}{\left(1-e^{-\rho(d) t}\right)}+\rho(d) t \log \lambda-\rho(d) t \log c \rho(d), & \text { if } y \geq 1-t \rho(d), \\
\infty & \text { if } y<1-t \rho(d),
\end{array}\right.
$$

where $\alpha=\alpha(y, t)$ uniquely solve the equation $\frac{1-e^{-\alpha}}{\alpha}=\frac{1-y}{\rho(d) t}$. 
(Doku-Amponsah,2014) obtained the same result for the proportion of detached nodes in $\mathcal{G}(n, n t / 2)$.

\section{Proof of the Results}

\subsection{Proof of Theorem 0.1 by Random Allocation}

We recall the conditional TRGG from Subsection 0.1 and denote by $\mathcal{X}\left(a_{1}\right)$ the collection of nodes which have type $a_{1} \in \mathcal{B}$.

Note that

$$
\sharp X\left(a_{1}\right)=n \varpi_{n}\left(a_{1}\right) .
$$

The random allocation model is appropriately obtained when typed balls are dropped at random in typed bins. In the next Lemma we prove the exponential equivalence for the distribution of $\tilde{\mathcal{M}}_{X}$, see (Doku-Amponsah, 2014), with respect to $\tilde{\mathbb{P}}_{\left(\varpi_{n}, \omega_{n}\right)}$ the law of the random allocation model and $\mathcal{M}$ with respect to $\mathbb{P}_{\left(\varpi_{n}, \omega_{n}\right)}$ where

$$
\mathbb{P}_{\left(\varpi_{n}, \omega_{n}\right)}=\mathbb{P}\left\{\cdot \mid \mathcal{H}\left(\mathcal{M}_{X}\right)=\left(\varpi_{n}, \omega_{n}\right)\right\}
$$

Thus the distribution of the TRGG conditioned to have type law $\varpi_{n}$ and edge distribution $\varpi_{n}$. Recall the definition of exponential equivalence, see (Dembo et al., 1998, Definition 4.2.10). We define the metric $\mathcal{D}$ of total variation by

$$
\mathcal{D}(\ell, \tilde{\ell})=\frac{1}{2} \sum_{\left(a_{1}, \sigma\right) \in \mathcal{B} \times \mathcal{Z}^{\mathcal{B}}}\left|\ell\left(a_{1}, \sigma\right)-\tilde{\ell}\left(a_{1}, \sigma\right)\right|, \quad \text { for } \ell, \tilde{\ell} \in \mathcal{P}\left(\mathcal{B} \times \mathcal{Z}^{\mathcal{B}}\right)
$$

and observe that this metric generates the weak topology.

Lemma 0.4. For every $\varepsilon>0$,

$$
\lim _{n \rightarrow \infty} \frac{1}{n} \log \mathbb{P}\left\{\mathcal{D}\left(\mathcal{M}_{X}, \tilde{\mathcal{M}}_{X}\right) \geq \varepsilon\right\}=-\infty,
$$

where $\mathbb{P}$ is a suitable coupling between the laws $\tilde{\mathbb{P}}_{\left(\varpi_{n}, \omega_{n}\right)}$ and $\mathbb{P}_{\left(\varpi_{n}, \omega_{n}\right)}$.

Proof. Proof of this Lemma given below is also uses the same coupling argument of (Boucheron et al., 2003) presented in (Doku-Amponsah, 2014). To begin, we label the bins $X_{1}, X_{2}, \ldots, X_{n}$ and denote by $Z\left(X_{i}\right), i=1,2,3, \ldots, n$ the types of the bins.

For each $a_{1}, a_{2} \in \mathcal{B}$ we begin coupling procedure as follows: At every step $u=1, \ldots, m_{n}\left(a_{1}, a_{2}\right)$, we pick at random two nodes $X_{i}^{u} \in \mathcal{X}\left(a_{1}\right)$ and $X_{j}^{u} \in \mathcal{X}\left(a_{2}\right)$. We place one ball of type $a_{2}$ into bin $X_{i}^{u}$ and one ball of type $a_{1}$ in $X_{j}^{u}$, and join $X_{i}^{u}$ with $X_{j}^{u}$ by an edge except when $X_{i}^{u}=X_{j}^{u}$ or the pair of nodes already formed an edge. If one of these three scenario occur, then we only pick two vertices at random from the set of all vertices of types $a_{1}$ and $a_{2}$, which are not already an edge in our graph model and we place an edge between them. This ends the formation of the graph having $\mathcal{L}_{X}^{1}=\varpi_{n}, \mathcal{L}_{X}^{2}=\omega_{n}$. For this collection denote, for each bin $X_{i} \in\left\{X_{1}, X_{2}, \ldots, X_{n}\right\}$, by $\sigma^{i}\left(a_{2}\right)$ the number of balls of type $a_{2} \in \mathcal{B}$ it contains, and define the empirical occupancy measure of the collection by

$$
\tilde{\mathcal{M}}_{X}\left(a_{1}, \sigma\right)=\frac{1}{n} \sum_{v \in V} \delta_{(\tilde{X}(v), \tilde{\mathcal{A}}(v))}\left(a_{1}, \sigma\right), \quad \text { for }\left(a_{1}, \sigma\right) \in \mathcal{B} \times \mathcal{Z}^{\mathcal{B}} .
$$

Observe that

$$
\mathcal{D}\left(\mathcal{M}_{X}, \tilde{\mathcal{M}}_{X}\right) \leq \frac{2}{n} \sum_{a_{1}, a_{2} \in \mathcal{B}} B^{n}\left(a_{1}, a_{2}\right)
$$

where $B^{n}\left(a_{1}, a_{2}\right)$ is the total number of steps $u \in\left\{1, \ldots, m_{n}\left(a_{1}, a_{2}\right)\right\}$ at which there is inconsistency between the nodes $X_{i}^{u}, X_{j}^{u}$ sampled and the nodes that received the $u^{\text {th }}$ edge linking $a_{1}$ and $a_{2}$ in the graph formation.

If $a_{1}, a_{2} \in \mathcal{B}$, the frequency of $X_{i}^{u}=X_{j}^{u}$ or the two nodes are already linked is equal to

$$
p_{[u]}\left(a_{1}, a_{2}\right) \leq \frac{1}{m_{n}\left(a_{1}, a_{2}\right)} \mathbb{1}_{\left\{a_{1}=a_{2}\right\}}+\left(1-\frac{1}{m_{n}\left(a_{1}, a_{2}\right)} \mathbb{1}_{\left\{a_{1}=a_{2}\right\}}\right) \frac{(u-1)}{\left(m_{n}\left(a_{1}, a_{2}\right)\right)^{2}} .
$$

$B^{n}\left(a_{1}, a_{2}\right)$ is a sum of independent 0 or 1 random variables $Y_{1}, \ldots, Y_{m_{n}\left(a_{1}, a_{2}\right)}$ with 'success' frequencies equal to $p_{[1]}\left(a_{1}, a_{2}\right), \ldots, p_{\left[m_{n}\left(a_{1}, a_{2}\right)\right]}\left(a_{1}, a_{2}\right)$. Note that $\mathbb{E}\left[Y_{u}\right]=p_{[u]}\left(a_{1}, a_{2}\right)$ and

$$
\operatorname{Var}\left[Y_{u}\right]=p_{[u]}\left(a_{1}, a_{2}\right)\left(1-p_{[u]}\left(a_{1}, a_{2}\right)\right)
$$


Now, we have

$$
\mathbb{E} B^{n}\left(a_{1}, a_{2}\right)=\sum_{k=1}^{m_{n}\left(a_{1}, a_{2}\right)} p_{[u]}\left(a_{1}, a_{2}\right) \leq \mathbb{1}_{\left\{a_{1}=a_{2}\right\}}+\left(1-\mathbb{1}_{\left\{a_{1}=a_{2}\right\}} \frac{1}{m_{n}\left(a_{1}, a_{2}\right)}\right)\left(1-\frac{1}{m_{n}\left(a_{1}, a_{2}\right)}\right) \leq 1+\mathbb{1}_{\left\{a_{1}=a_{2}\right\}} .
$$

We write

$$
\sigma_{n}^{2}\left(a_{1}, a_{2}\right):=\frac{1}{m_{n}\left(a_{1}, a_{2}\right)} \sum_{k=1}^{m_{n}\left(a_{1}, a_{2}\right)} \operatorname{Var}\left[Y_{k}\right]
$$

and observe that

$$
\lim _{n \rightarrow \infty} \mathbb{E}\left(B^{n}\left(a_{1}, a_{2}\right)\right)=\lim _{n \rightarrow \infty} \operatorname{Var}\left(B^{n}\left(a_{1}, a_{2}\right)\right)=\lim _{n \rightarrow \infty} m_{n}\left(a_{1}, a_{2}\right) \sigma_{n}^{2}\left(a_{1}, a_{2}\right)=\mathbb{1}_{\left\{a_{1}=a_{2}\right\}}+1 .
$$

Define $h(t)=(1+t) \log (1+t)-t$, for $t \geq 0$ and apply Bennett's inequality, see ( Bennett, 2004) to obtain, for very large $n$

$$
\mathbb{P}\left\{\frac{B^{n}\left(a_{1}, a_{2}\right)}{n} \geq \frac{\mathbb{1}_{\left\{a_{1}=a_{2}\right\}}+1}{n}+\delta_{1}\right\} \leq \exp \left[-m_{n}\left(a_{1}, a_{2}\right) \sigma_{n}^{2}\left(a_{1}, a_{2}\right) h\left(\frac{n \delta_{1}}{n\left(a_{1}, a_{2}\right) \sigma_{n}^{2}\left(a_{1}, a_{2}\right)}\right)\right],
$$

for any $\delta_{1}>0$. Let $\varepsilon \geq 0$ and choose $\delta_{1}=\frac{\varepsilon}{2 m^{2}}$. Suppose that we have $B^{n}\left(a_{1}, a_{2}\right) \leq \delta$. Then, by (6),

$$
d\left(\mathcal{M}_{X}, \ell_{n}\right) \leq 2 \delta_{1} m^{2}=\varepsilon
$$

Hence,

$$
\begin{aligned}
\mathbb{P}\left\{\mathcal{D}\left(\mathcal{M}_{X}, \tilde{\mathcal{M}}_{X}\right)>\varepsilon\right\} \leq \sum_{a_{1}, a_{2} \in \mathcal{B}} \mathbb{P}\left\{B^{n}\left(a_{1}, a_{2}\right) \geq n \delta_{1}\right\} & \leq m^{2} \sup _{a_{1}, a_{2} \in \mathcal{B}} \mathbb{P}\left\{B^{n}\left(a_{1}, a_{2}\right) \geq \mathbb{1}_{\left\{a_{1}=a_{2}\right\}}+1+\left(n \delta_{1}\right) / 2\right\} \\
& \leq m^{2} \sup _{a_{1}, a_{2} \in \mathcal{B}} \exp \left[-m_{n}\left(a_{1}, a_{2}\right) \sigma_{n}^{2}\left(a_{1}, a_{2}\right) h\left(\frac{n \delta_{1}}{m_{n}\left(a_{1}, a_{2}\right) \sigma_{n}^{2}\left(a_{1}, a_{2}\right)}\right)\right] .
\end{aligned}
$$

Let $0 \leq \delta_{2} \leq 1$. The, for very large $n$ we that have

$$
\begin{aligned}
& \frac{1}{n} \log \mathbb{P}\left\{\mathcal{D}\left(\mathcal{M}_{X}, \tilde{\mathcal{M}}_{X}\right)>\varepsilon\right\} \leq-\left(1-\delta_{2}\right) h\left(\frac{n \delta_{1}}{2\left(1+\delta_{2}\right)}\right) \\
& \quad=-\left(\mathbb{1}_{\left\{a_{1}=a_{2}\right\}}+1-\delta_{2}\right)\left[\left(\frac{1}{n}+\frac{\delta_{1}}{2\left(\mathbb{1}_{\left\{a_{1}=a_{2}\right\}}+1+\delta_{2}\right)}\right) \log \left(1+\frac{n \delta_{1}}{2\left(\mathbb{1}_{\left\{a_{1}=a_{2}\right\}}+1+\delta_{2}\right)}\right)-\frac{\delta_{1}}{2\left(\mathbb{1}_{\left\{a_{1}=a_{2}\right\}}+1+\delta_{2}\right)}\right] .
\end{aligned}
$$

This ends the proof of the Lemma.

To conclude the proof of Theorem 0.1 , we note that empirical occupancy measure $\tilde{\mathcal{M}}_{X}$ is exponential equivalent to $\mathcal{M}_{X}$, and further $\tilde{\mathcal{M}}_{X}$ under the law $\tilde{\mathbb{P}}_{\left(\varpi_{n}, \omega_{n}\right)}$ obeys a large deviation principle with rate function $\tilde{J}_{((\varpi, \omega)}$ by (Doku-Amponsah, Lemma 5, 2014). Therefore, by the exponential equivalent theorem, see (Dembo et al.,1998, Theorem 4.2.13), $M$ obeys an LDP with the rate function $\tilde{J}_{((\varpi, \omega)}$.

\subsection{Proof of Corollary 0.3 by the Contraction Principle}

We prove Corollary 0.3 from Theorem 0.2 by using the contraction principle, (?, Theorem 4.2 .1$)$ on the linear mapping $G: \mathcal{P}(\mathcal{Z}) \rightarrow[0,1]$ given by $G(\delta)=\delta(0)$. To be specific, Theorem 0.2 implies an LDP for random variable $G\left(D_{X}\right)=D_{X}(0)$ with good rate function

$$
\xi(y)=\inf \left\{H\left(\delta \| q_{\langle\delta\rangle}\right): \delta \in \mathcal{P}(\mathcal{Z}), \delta(0)=y, \sum_{r=0}^{\infty} r \delta(r)=\rho(d) t\right\} .
$$

Note that, we have

$$
\rho(d) t=\sum_{k=1}^{\infty} k \delta(k) \geq \sum_{k=1}^{\infty} \delta(k)=1-y
$$

and that the class of measures satisfying the two constraints is necessarily empty if $t \rho(d)<1-y$. If $\rho(d) t \geq$ $1-y$,using the Lagrangian method we can calculate the minimizer $p$, defined by $p(0)=y, p(k):=U(y, t)^{-1} \frac{(\alpha(y, t))^{k}}{k !}$ where $\alpha(y, c)$ uniquely solve

$$
\frac{e^{\lambda}-1}{\lambda}=\frac{1-y}{\rho(d) t}
$$


and $U(y, t):=\frac{e^{\lambda-1}}{1-y}$. Therefore, we have

$$
\begin{aligned}
\eta(y) & =x \log \frac{y}{q_{c \rho(d)}(0)}+(1-y) \log \frac{(1-y)}{1-q_{c \rho(d)}(0)}+(1-y) \sum_{k=1}^{\infty} p(k) \log \frac{p(k)}{\hat{q}_{\rho(d) t}(k)} . \\
& =y \log \frac{y}{q_{\rho(d) t}(0)}+(1-y) \log \frac{(1-y)}{1-q_{t \rho(d)}(0)}+t \rho(d) \log \frac{\lambda}{t \rho(d)} \\
& =y \log \frac{y}{e^{-\rho(d) t}}+(1-y) \log \frac{(1-y)}{1-e^{-\rho(d) t}}+t \rho(d) \log \frac{\lambda}{t \rho(d)}
\end{aligned}
$$

if $t \rho(d) \geq 1-y$ and $\infty$ otherwise. Particularly, if $y=e^{-t \rho(d)}$ then we have $\alpha(y, t)=t \rho(d)$, which gives $\eta\left(e^{-t \rho(d)}\right)=0$. This end the proof of Theorem 0.3.

\section{Acknowledgements}

We are thankful to the referees for their suggestions which have helped to improve this article.

\section{References}

Biggins, J. D., \& Penman, D. B. (2009). Large deviations in randomly coloured random graphs Electron. Comm. Probab., 14, 290-301. http://dx.doi.org/10.1214/ECP.v14-1478

Boucheron, S., Bousquet,O., \& Lugosi, G. (2004). Concentration inequalities. In: Advanced lectures in machine learning, Eds: O. Bousquet, U. v. Luxburg and G. Rötsch. Springer 208-240.

Cannings, C., \& Penman, D. B. (2003). Models of random graphs and their applications. In: Handbook of Statistics 21. Stochastic Processes: Modelling and Simulation. Eds: D.N. Shanbhag and C. R. Rao. Elsevier 51-91.

Dembo, A., \& Zeitouni, O. (1998). Large deviations techniques and applications. Springer, New York.

Doku-Amponsah, K. (2006). Large deviations and basic information theory for hierarchical and networked data structures. PhD Thesis, Bath.

Doku-Amponsah, K. (2014). Exponential approximation, method of types for empirical neighbourhood distributions of random graphs by random allocation. International Journal of Statistics and Probability, 3(2), 110-120. http://dx.doi.org/10.5539/ijsp.v3n2p110

Doku-Amponsah, K. (2014[b]). Joint large deviation principle for empirical measures for near intermediate random geometric graphs. Unpublished.

Doku-Amponsah, K., \& Mörters, P. (2010). Large Deviation Principles for empirical Measures of Coloured random graphs. The annals of Applied Probab., 20(6), 1989-2021. http://dx.doi.org/10.1214/09-AAP47

Feller, W. (1967). An introduction to probability theory and its applications. Volume I. Wiley, New York. Third edition.

MCDiarmid, C. J. H., \& Müller, T. (2005). Colouring random geometric graphs. DMTCS proc. AE, 1-4.

O'Connell, N. (1988). Some large deviation results for sparse random graphs. Probab. Theory Relat. Fields, 110, 277-285. http://dx.doi.org/10.1007/s004400050149

Penman, D. B. (1998). Random graphs with correlation structure. PhD Thesis, Sheffield.

Penrose, M. D. (2003). Random geometric Graphs. Oxford University press. Oxford.

\section{Copyrights}

Copyright for this article is retained by the author(s), with first publication rights granted to the journal.

This is an open-access article distributed under the terms and conditions of the Creative Commons Attribution license (http://creativecommons.org/licenses/by/3.0/). 